Communications in Physics, Vol.29, No. 1 (2019), pp. 47-54

DOI:10.15625/0868-3166/29/1/13221

\title{
CHARACTERIZATION OF HIGH-POWER DFB-MOPA DIODE LASERS EMITTING AT 1064 nm
}

\author{
THANH-PHUONG NGUYEN ${ }^{\dagger}$
}

School of Engineering Physics, Hanoi University of Science and Technology, No. 1 Dai Co Viet, Hai Ba Trung, Hanoi, Vietnam

${ }^{\dagger}$ E-mail: phuong.nguyenthanh@hust.edu.vn

Received 23 October 2018

Accepted for publication 29 December 2018

Published 31 January 2019

\begin{abstract}
Electro-Optical spectral characteristics and beam quality of the high power monolithic DFB-MOPA lasers emitting at $1064 \mathrm{~nm}$ have been investigated at the room temperature as function of injection current. Laser beam quality is characterized by waist diameter and far-field divergence angle versus average optical output power. Beam propagation ratio $M^{2}$ is defined at difference intensity levels from lateral beam profile giving more detail laser behaviors at high power.
\end{abstract}

Keywords: high power semiconductor lasers, DFB-MOPA, beam quality.

Classification numbers: 42.55.Px; 42.60.By; 42.60.Jf.

\section{INTRODUCTION}

High-power pulsed lasers emitting at $1064 \mathrm{~nm}$ have been requested for many applications such as health-care [1], material processing [2], free-space measurement, environmental research [3-6]. Especially, in the remote sensing technique for detection of important components of the atmosphere or distance measurements in LIght Detection And Ranging (LIDAR), the 1064 nm laser sources play an important role because of their short pulse duration and high power delivery. Solid state lasers based on $\mathrm{Nd}^{3+}$ doped crystals are good candidate for such applications [3]. However, in the aspect of compactness, many applications need small size and non-complex laser systems. Therefore, diode lasers and, in particular, DFB-MOPA diode lasers which have been developed recently $[7,8]$ seem to be suitable substitution. In these reports, DFB-MOPA diode laser characteristics were demonstrated to nearly satisfy the demands for an expected solid-state laser: small size, high pulse repetition rate, high optical output power, and narrow optical spectrum.

(C)2019 Vietnam Academy of Science and Technology 
Hence, applications based-on high-power diode lasers have been more attractive and promissing when flexible and compact device system such as portable and mobile equipment can be designed.

In this report, the high power $1064 \mathrm{~nm}$ monolithic DFB-MOPA diode laser, which possesses a $4 \mathrm{~mm}$ amplifier and a high average output power reachable up to $6 \mathrm{~W}$ has been investigated at the room temperature. The optical spectra are studied as function of the injection current driven up to $10 \mathrm{~A}$. Beam quality of the DFB-MOPA lasers that is defined by beam propagation ratio $\mathrm{M}^{2}$ has been, for the first time, characterized versus average optical output power. Similarly, the relevant beam parameters such as waist diameter and far-field divergence are determined at difference intensity levels giving detail information about beam profile, which is quite a necessity to the users to decide how to couple the laser beam into fibers and focus it to optical systems.

\section{EXPERIMENT}

\section{Laser structure}

The DFBMOPA diode laser under this research consists of a $1 \mathrm{~mm}$ index-guided DFB ridge-waveguide (RW) laser, a $1 \mathrm{~mm}$ picker and a $4 \mathrm{~mm}$ gain-guided tapered amplifier (Fig. 1) DFB laser is operated in continuous mode as Master Oscillator (MO). The picker and amplifier are worked in pulse mode as Power Amplifier (PA). Both sections are separately contacted and can be driven independently.

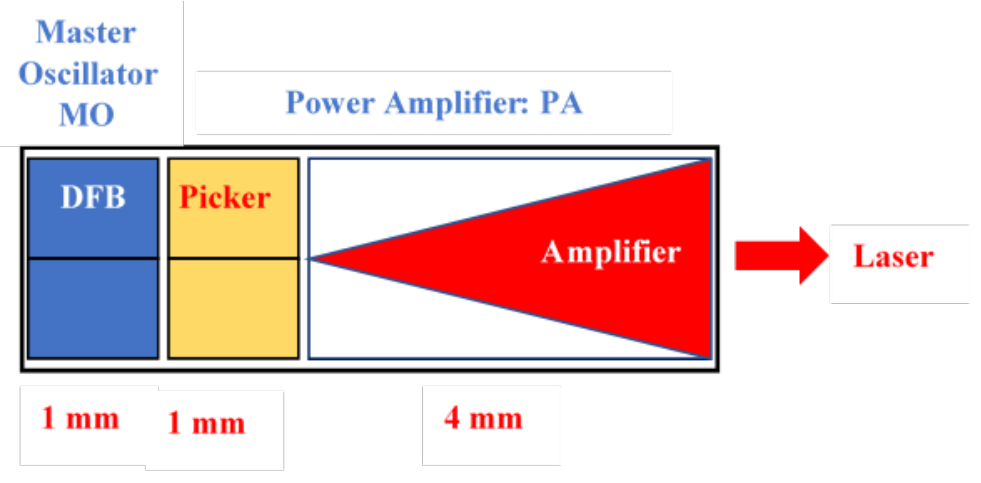

Fig. 1. Schematic view of a DFB-MOPA diode laser with the total length of $6 \mathrm{~mm}$.

DFB lasers are grown by low-pressure metal organic vapor phase epitaxy (MOVPE) base on GaAs in two steps. An InGaAs active double quantum well (QW) layer is embedded in an AlGaAs waveguide. A second order grating with a period of about $320 \mathrm{~nm}$ is formed by holographic photolithography and wet-chemical etching. Details of the DFB-laser structure are described [9]. The $1 \mathrm{~mm}$ picker is operated in pulse mode to transform the CW operation of the DFB laser into optical pulses. When no current injects into this section, it acts as an optical gate and absorbs the coupled CW beam of the DFB laser. When injected by current pulse, the picker becomes transparent and the DFB laser beam can pass.

A laser diode controller Newport 525B provides injection current for the DFB laser with a fixed current of $150 \mathrm{~mA}$. The PA part is driven by a pulse of current source DEI PCX-7410 that can generate square wave pulse signals from 0 to $10 \mathrm{~A}$. Pulse repetition frequency is chosen of 
$1 \mathrm{kHz}$ and pulse width value is $1 \mu \mathrm{s}$. A Newport Temperature Controller TED350 stabilizes the laser mount temperature. The laser heat sink is kept at constant temperature of $25^{\circ} \mathrm{C}$ using a water circulator. The average power versus pulse currents is measured by a fast photo-diode New Focus 1434 and displayed by a Gentec power meter. The bias voltage is measured by an oscilloscope Tektronix TDS 410 A. An Advantest optical spectrum analyzer model Q8384 is used providing a spectral resolution in order of ten pm and a dynamic range of $60 \mathrm{~dB}$.

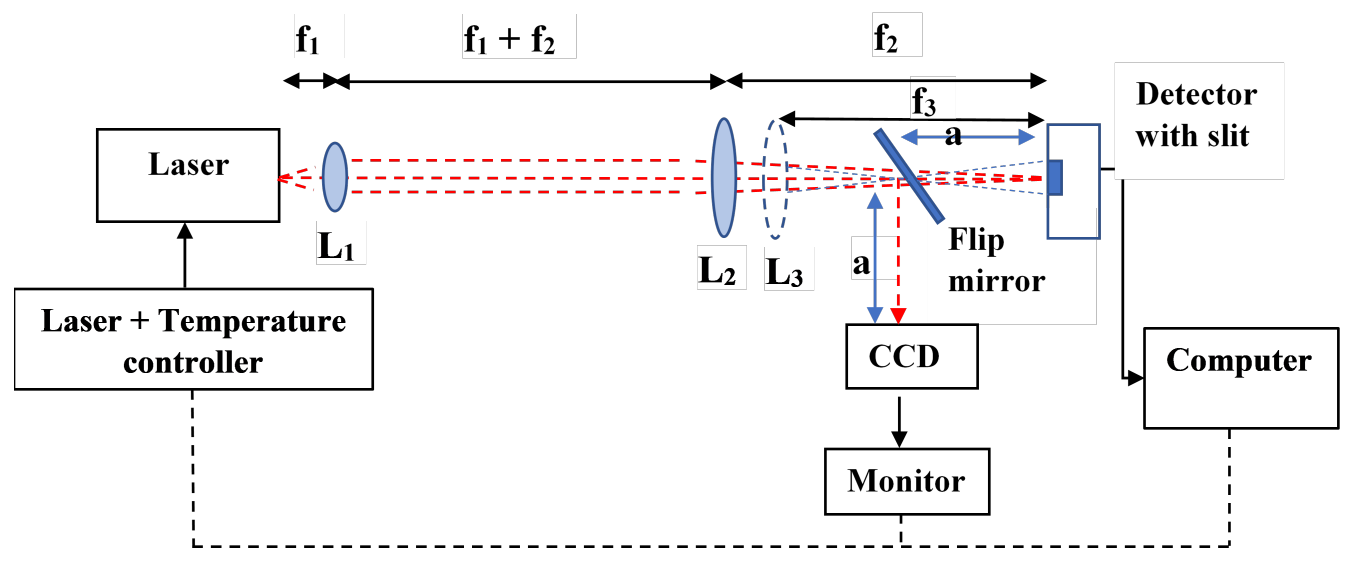

Fig. 2. Principle beam quality experimental setup. $L_{1}$ : Collimation lens; $L_{2}$ : Focus telescope lens; $L_{3}$ : Additional focus lens.

Figure 2 presents the principle experimental setup for the determination of laser beam quality. The methods to measure beam gauges in the form of the beam propagation factor $\mathrm{M}^{2}$ are specified in the standard DIN EN ISO 11146 [10]. In this setup, the diode laser can be moved back and forth along the optical axis around the focal point of the lens $L_{1}$. In the fast axis direction the waist of laser beam is located near the facet of the active layer (near-field), while the beam waist in the slow axis direction is located somewhere behind (far-field). The distance between these two positions is the astigmatism. The laser beam is collimated by a lens $L_{1}$ and focused to a detector by a telescope lens $L_{2}$. A flip mirror reflects laser signal to a CCD camera. A monitor connected to the CCD camera is used to observe and control the signal. When the beam profile can be seen as focused spot clearly in the fast axis direction on the monitor, the mirror is taken out, the near-field profiles are recorded by a detector with slit. The slit width is set in this case a value of $5 \mu \mathrm{m}$. The detector is moved step-by-step to sweep all image. After that, the laser is moved, when the beam profile can be seen as focused spot in the slow axis direction, the beam waist profile in the slow axis is recorded. A lens $L_{3}$ is added in the system. The far-field full divergence angle is given by the beam diameter in the focal plane of the lens $L_{3}$.

\section{RESULTS AND DISCUSSIONS}

Figure 3 presents PUI characteristics of the $1064 \mathrm{~nm}$ DFB-MOPA diode laser. The DFB laser was supplied with CW current of $150 \mathrm{~mA}$ and the temperature of the heat sink was kept at $25^{\circ} \mathrm{C}$. The redsolid line presents average optical output power versus pulse injection current. The 
pumping current to PA part was varied from 0 to $10 \mathrm{~A}$. The laser has a threshold current of about $1850 \mathrm{~mA}$. At the injection current of $10 \mathrm{~A}$, average optical output power reached a value of 6 $\mathrm{W}$. Therefore, a slope efficiency $\Delta P / \Delta I$ of about $0.75 \mathrm{~W} / \mathrm{A}$ remains constant when the injection current was diving up to $10 \mathrm{~A}$. Voltage of $3.3 \mathrm{~V}$ drops across the device at maximum pumping current (dotted-black line). The conversion efficiency is represented by dashed-blue curve line and a maximum conversion efficiency is about $20 \%$ corresponds with (Fig. 3).

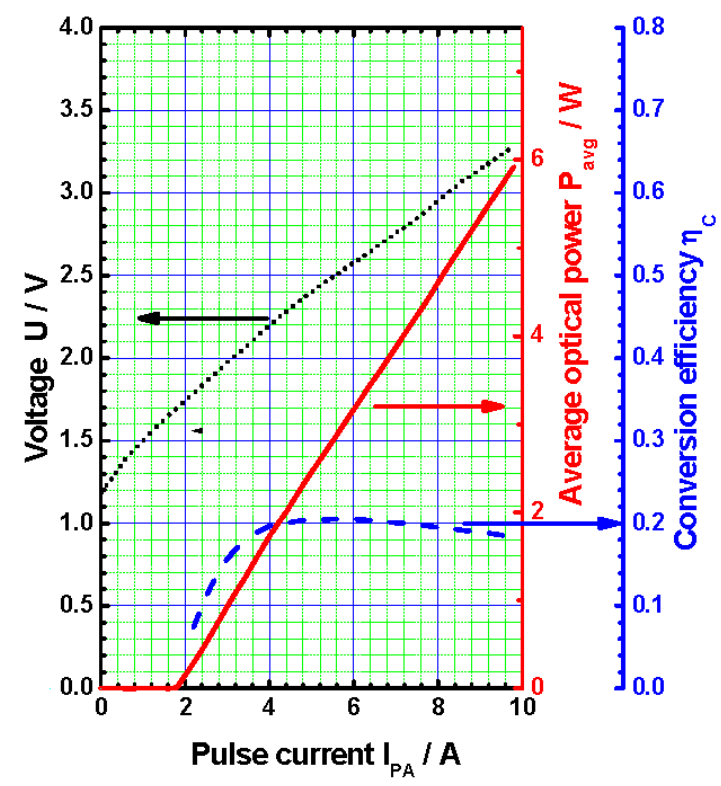

Fig. 3. Average optical output power of the $1064 \mathrm{~nm}$ DFB-MOPA laser as a function of the pulse injection current to the PA when fixed injection currents is $150 \mathrm{~mA}$ to the $\mathrm{MO}$ and heat sink temperature is $25^{\circ} \mathrm{C}$.

Fig. 4 presents optical spectrum of the DFB-MOPA laser at fixed MO current of $150 \mathrm{~mA}$ and the PA current of $8.6 \mathrm{~A}$ (corresponding to an average optical output power of $5 \mathrm{~W}$ ). The graph shows a single lateral lasing mode at $1063.47 \mathrm{~nm}$. Side mode suppression ratio exceeds $35 \mathrm{~dB}$ and full width half maximum is $0.16 \mathrm{~nm}$.

Fig. 5 shows the optical spectra of the DFB-MOPA diode laser as function of the injection current of the PA part. The pulse current supplied to PA part from 0 A to 10 A with step of 0.2 A. Spectra were recorded for each injection current setting and analyzed with the Origin program. The corresponding data are displayed in a 3D-graph, with the axes of wavelength, current and relative intensity as a spectral map show in Fig. 5. The relative intensity measured in linear scale that presents the center wavelength of the DFB-MOPA does not depend on injection current. That property can be explained from structure of the lasers In this case, the narrow waveguiding MO part is responsible for the selection of a single lateral lasing mode. The center wavelength depends strongly on injection current and temperature of the DFB [11], however, when the the DFB injection current was fixed at $150 \mathrm{~mA}$ and heat sink temperature was at $25^{\circ} \mathrm{C}$, DFB laser wavelength 


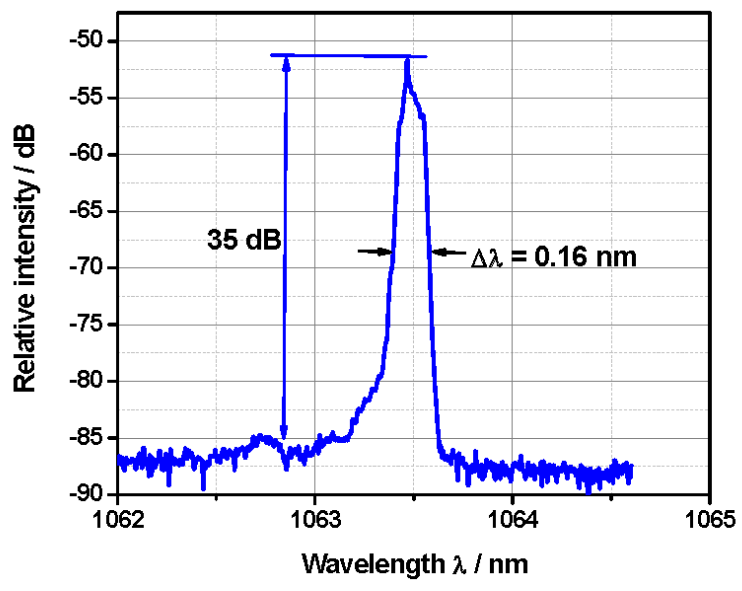

Fig. 4. Optical spectrum of the DFB-MOPA diode laser emitting around $1064 \mathrm{~nm}$ at $I_{D F B}=150 \mathrm{~mA}, T=25^{\circ} \mathrm{C}$, and $P=5 \mathrm{~W}$.

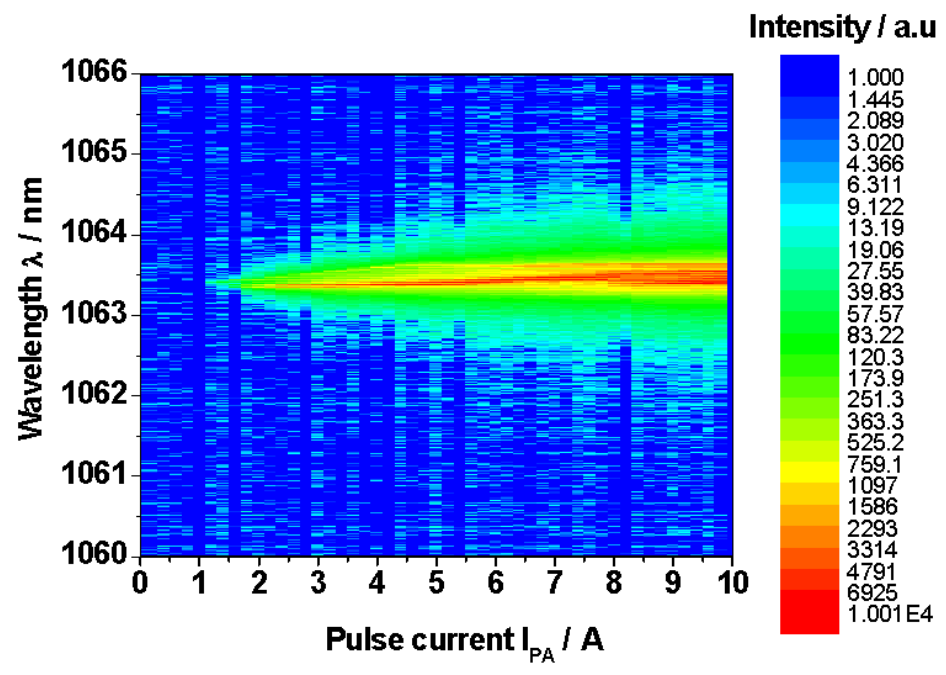

Fig. 5. Spectral map of $1064 \mathrm{~nm}$ DFB-MOPA laser @ $\mathrm{I}_{D F B}=150 \mathrm{~mA}, T=25^{\circ} \mathrm{C}$.

does not change. The results in Fig. 5 show the wavelength of the $1064 \mathrm{~nm}$ DFB-MOPA is independent on injection current to PA part. Hence, the DFB-MOPA laser can operate up to $6 \mathrm{~W}$ with stable frequency

Figure 6 presents the lateral intensity profiles at an average output power of $5 \mathrm{~W}$ of the DFBMOPA diode laser. The near-field position, the beam-waist position, and the far-field angle of the slow axis are shown. From the profile, beam propagation ratios are calculated following $[12,13]$ :

$$
M 2=\frac{w_{0} \Phi \pi}{4 \lambda}
$$


where the position of the beam waist is known, and its intensity profile is readily accessible for measurement, then the beam waist diameter $\left(w_{0}\right)$ and the far-field full divergence angle $(\Phi)$, along with the wavelength $(\lambda)$, are used to infer the beam propagation product. The values of $w_{0}$ and $\Phi$ are determined at $1 / \mathrm{e}^{2}$ intensity levels, at $95 \%$ intensity levels or at the position that intensity distribution is calculated with the help of the second order moment give different beam propagation ratios.
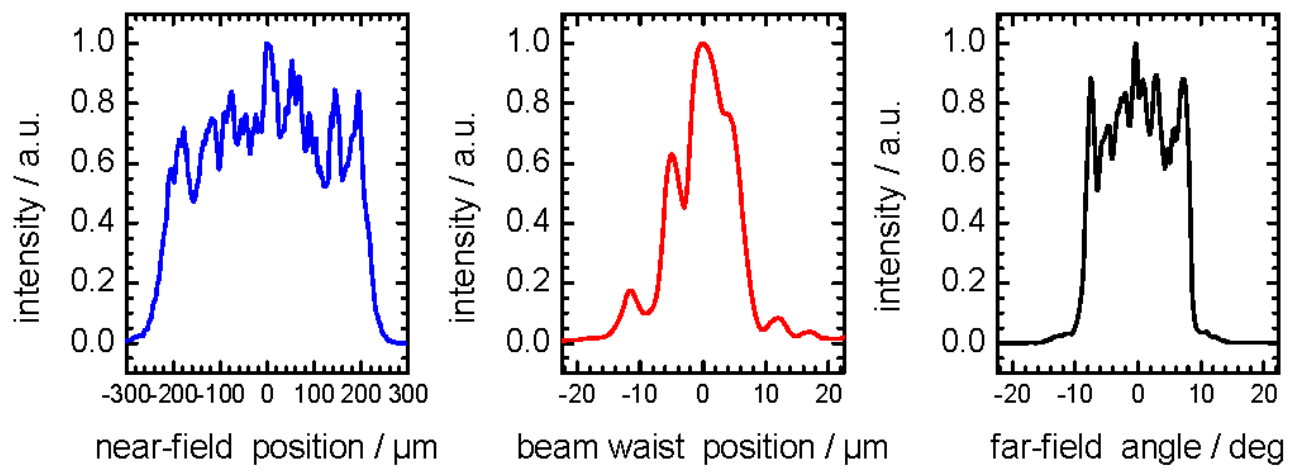

Fig. 6. Lateral intensity profiles at average output power of $5 \mathrm{~W}$ in the near-field, in the beam-waist, and in the far-field of $1064 \mathrm{~nm}$ DFB-MOPA laser at the fixed injection currents of $150 \mathrm{~mA}$ to the MO.

At the average optical output power of $5 \mathrm{~W}$ as shows in Fig. 6, the beam propagation ratios get the values of $M_{1 / e^{2}}^{2}=4.7, M_{95 \%}^{2}=7.3$, and $M_{2 . m o m}^{2}=7.9$. The power content profile is integrated from beam profile starting at the center of gravity, until the sum reaches where the intensity drops to $1 / \mathrm{e}^{2}$ of the maximum intensity, $P_{C L}=68 \%$.

Beam properties of the $1064 \mathrm{~nm}$ DFB-MOPA laser are characterized as function of average optical output power, as shown in Fig. 7. In the case, average optical output power was established from 1 to $6 \mathrm{~W}$ (with step of $1 \mathrm{~W}$ ) and at every output power the lateral intensity profiles and the interested parameters were recorded.

The beam waist is expanded at high power, so that the power content profile at $1 / \mathrm{e}^{2}$ levels is decreased from $P_{C L}=83 \%$ to $P_{C L}=64 \%$ (Fig. 7a). In this case, the whole MOPA works as a compound cavity, where the PA section acts as an active feedback section. In higher power, the PA gives more feedback to the MO part and strong intensity variations cause spatial hole burning of carriers within the PA section. Besides, when the injection current to the PA is increased, the lateral beam profile suffers degradations and/or higher order modes. Consequent is oscillation due to of non-linear effects such as thermal lensing, carrier-induced index suppression $[14,15]$.

The lateral far-field full divergence angle is hardly changed, the values are approximate $17^{\circ}$ at $95 \%$ and $16^{\circ}$ at $1 / \mathrm{e}^{2}$ of intensity profile (Fig. $7 \mathrm{~b}$ ). The far-field angle of the $1064 \mathrm{~nm}$ DFB-MOPA laser does not depend on average optical output power up to $6 \mathrm{~W}$.

The full width of the beam waits $w_{0}$ increases clearly when the output power $P_{P A}$ changes from $1 \mathrm{~W}$ to $6 \mathrm{~W}$, therefore the value of $M^{2}$ are larger at higher output power (Fig. $7 \mathrm{c}$ ). The $M_{1 / e^{2}}^{2}$ 

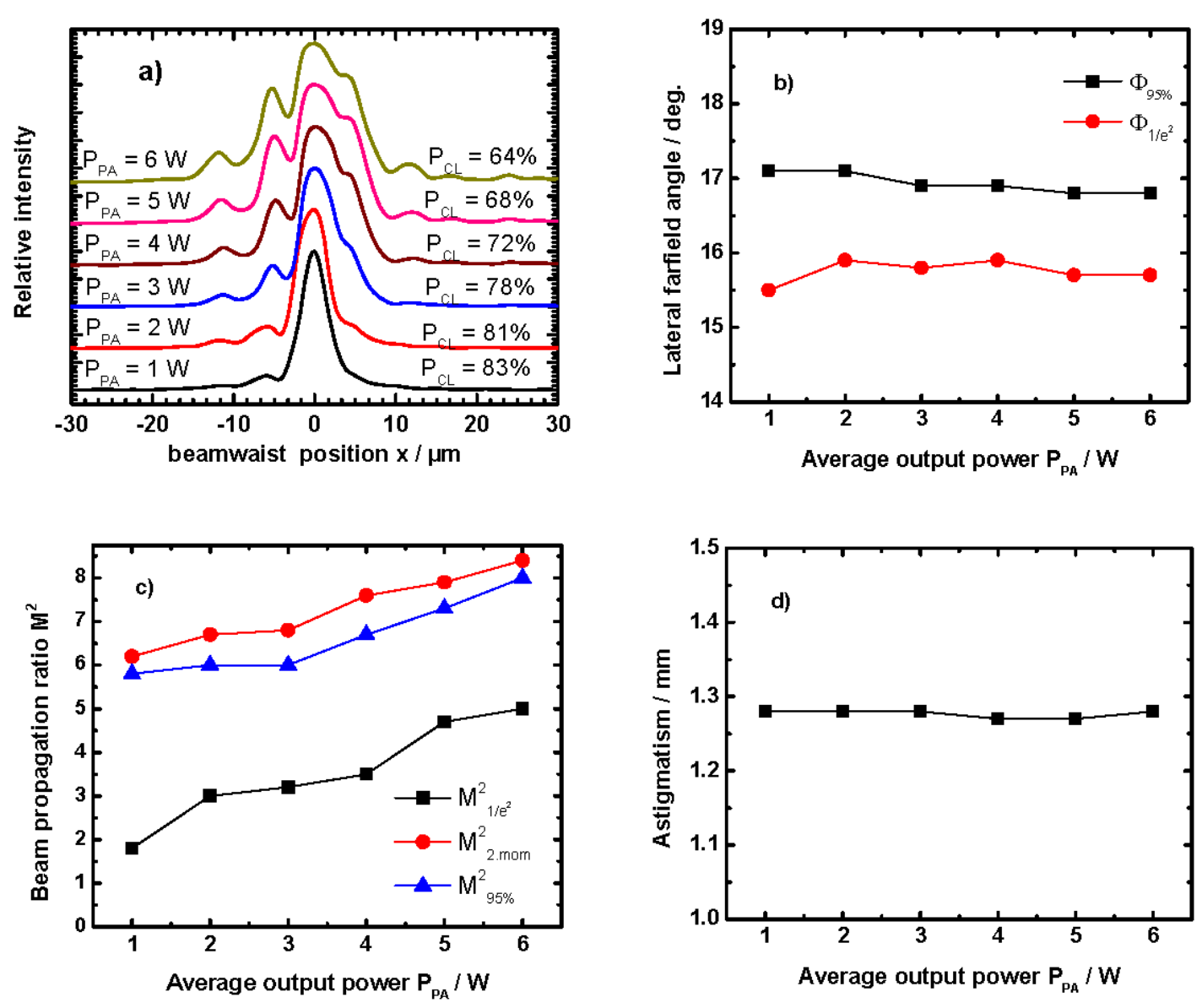

Fig. 7. Lateral beam waist (a), far-field angle at $95 \%$ and $1 / \mathrm{e}^{2}$ levels (b), beam propagation ratios (c), and astigmatism (d) versus. average optical output power of the $1064 \mathrm{~nm}$ DFB-MOPA laser at the fixed injection currents of $150 \mathrm{~mA}$ to the MO part.

increases from 1.8 to 5 , while $M_{95 \%}^{2}$ is various from 5.8 to 8 . In the second moment mode, beam propagation ratio gets the largest value of 8.4 at average output power of $6 \mathrm{~W}$.

Fig. $7 \mathrm{~d}$ shows that the astigmatism of the laser hardly depends on optical output power. Astigmatism is $1.27 \mathrm{~mm}$ at average optical output power of $4 \mathrm{~W}$ and $5 \mathrm{~W}$, while at other power level the value is $1.28 \mathrm{~mm}$.

\section{CONCLUSIONS}

Characterizations of the $1064 \mathrm{~nm}$ DFB-MOPA diode laser have been done at the room temperature. The average optical output power reaches $6 \mathrm{~W}$ at a pulse injection current of $10 \mathrm{~A}$ to the PA part and a fixed DFB current of $150 \mathrm{~mA}$. The constant slope efficiency $\Delta \mathrm{P} / \Delta \mathrm{I}$ is about 0.75 W/A and a maximum conversion efficiency is about $20 \%$. The lasers give single lateral lasing mode at $1063.47 \mathrm{~nm}$. Side mode suppression ratio exceeds $35 \mathrm{~dB}$ and full width half maximum is 
$0.16 \mathrm{~nm}$. The wavelength of the $1064 \mathrm{~nm}$ DFB-MOPA laser is independent on injection current to PA part. The lasers can operate up to $6 \mathrm{~W}$ with stable frequency. Beam profile of the DFBMOPA laser was investigated carefully as function of average optical output power. The farfield divergence angle does not change when the optical output power increases. Beam waist is expanded at high power because inhomogeneous effects of laser material increase at high current density pumping applied in the PA section, then beam propagation ratios deteriorate consequently. These experimentally obtained results show convincingly not only the advantages of the DFBMOPA diode lasers as very compact, high power and fidelity, pulsed solid-state lasers, but also their limitation in device performance and laser material quality to be improved.

\section{ACKNOWLEDGMENTS}

The author would like to acknowledge support from Ferdinand-Braun-Institut for this research.

\section{REFERENCES}

[1] Y. Saydjari, T. Kuypers and N. Gutknecht, BioMed Research International 2016 (2016) 8421656.

[2] Z. Sun and C. M. Gupta, Appl. Surf. Sci. 456 (2018) 342.

[3] Ma. McGill, D. Hlavka, W. Hart, V. S. Scott, J. Spinhirne and B. Schmid, Appl. Opt. 41 (2002) 3725.

[4] D. R. Potter, Analytical devices for the measurement of water vapor in the natural gas process and transmission industry, GAS2011, Rotterdam (2011).

[5] C. Weitkamp, Lidar: range resolved optical remote sensing of the atmosphere, Springer series in optical science, 2005, pp. 252-253.

[6] A. Deleva and I. Grigorov, International Journal of Navigation and Observation 2011 (2011) 769264.

[7] T. N. Vu, A. Klehr, B. Sumpf, H. Wenzel, G. Erbert and G. Tränkle, Semicond. Sci. Technol. 29 (2014) 035012.

[8] O. Brox, F. Bugge, A. Ginolas, A. Klehr, P. Ressel, H. Wenzel, G. Erbert and G. Tränkle, High-power ridge waveguide DFB and DFB-MOPA lasers at $1064 \mathrm{~nm}$ with a vertical farfields angle of $15^{\circ}$, Novel In-Plane Semiconductor Lasers IX, 7616 (2010) 761610. International Society for Optics and Photonics, 2010. 7616 (2010) 761610.

[9] H. Wenzel, A. Klehr, M. Braun, F. Bugge, G. Erbert, J. Fricke and A. Knauer, Design and realization of highpower DFB lasers, Physics and Applications of Optoelectronic Devices, 5594 (2004) 110.

[10] ISO/FDIS 11146-1, ISO/FDIS 11146-2, ISO/TR 11146-3, Laser and laser-related equipment-Test methods for laser beam widths, divergence angles and beam propagation ratios, Deutsches Institut fur Normung e.V, 2004.

[11] T-P. Nguyen and T. Q. Tien, Influence of Resonator Parameters and Thermal Effect on Optical Spectra of Integrated Grating High Power Diode Lasers, Proceedings of the VII ${ }^{\text {th }}$ Conference on Photonics and Applications: Advances in Optics Photonics Spectroscopy \& Applications VII, ISSN-1859-4271 (2013) p. 904-909.

[12] N. Reng and B. Eppich, Opt. Quant. Electron. 24 (1992) 973.

[13] H. Sun, A Practical Guide to Handling Laser Diode Beams, SpringerBriefs in Physics, Springer, 2015.

[14] L. Goldberg, D. Mehuys, M. R. Surette and D. C. Hall, IEEE J. Quant. Electron. 29 (1993) 2028.

[15] F. R. Nash, J. Appl. Phys. 44 (1973) 4696. 